\title{
Proceedings of the inaugural meeting of the British Neurosurgery Research Group, Newcastle-upon-Tyne, United Kingdom, 31 March 1994
}

ROC CURVES: A NEW WAY OF ANALYSING NEUROSURGICAL DATA

MS Choksey, AD Mendelow, AR Chambers, A McNeigh. Department of Neurosurgery, Coventry, UK and Regional Neuroscience Centre, Newcastle-uponTyne, UK

A frequent problem encountered in neurosurgical studies is investigating the association between a continuous variable (CPP, ICP, doppler velocity, thermal clearance, $\mathrm{PO}_{2}$, haematoma volume) and a "yes/no" clinical state (outcome good/bad, ischaemia present/absent, deterioration occurred/did not occur). Traditionally, the approach has been to divide the continuous variable data into bands. This imposes constraints, as the bands are arbitrary, too many bands reduce the number of data in each, and too few make the dissection of a threshold value difficult.

Receiving operator characteristics (ROC) curves were developed in the second world war as a means of assessing the efficacy of radar and sonar operators. Graded echoes, increasing in size and mixed with background noise, were presented to different operators at receiving stations. Considerable differences in behaviour were seen between operators: however, individual operators were remarkably internally consistent. Their "characteristic behaviour" was plotted as an ROC curve: the sensitivity $v$ (1-specificity). These curves replaced former labels such as "brilliant", "pretty reliable", "cries wolf", "overcautious", "blind as a bat", and "absolutely useless".

Similar analysis can be used for neurosurgical data. A series of $2 \times 2$ hypotheses are created for each value of the parameter, and compared to the "yes/no" question. An association between the value of the continuous variable and the "yes/no" question is readily demonstrated by the shape of the curve and its distance from the diagonal. Additionally, the data can be plotted as a "likelihood ratio", which is simply the sensitivity/1-specificity plotted against increasing values of the continuous variable.

This method of analysis can easily be achieved with standard computers. It obviates the need for "banding" the continuous variable data and readily demonstrates a "pivotal" or threshold value above or below which the clinical state may change.

INTRACRANIAL REPAIR OF THE FACIAL NERVE USING A FREEZE-THAWED SKELETAL MUSCLE GRAFT

MG O'Sullivan, IR Whittle, M Glasby.

Departments of Neurosurgery and

Anatomy, University of Edinburgh, UK

Facial nerve injury occurs not infrequently during surgery in the cerebellopontine angle
(CPA). A novel method of facial nerve repair in the CPA using freeze-thawed skeletal muscle as a nerve graft is reported.

A posterior fossa craniectomy was performed in six adult sheep. The facial nerve was divided in the CPA and a freeze-thawed skeletal muscle interposition graft $^{1}$ was inserted and secured with fibrin glue. The animals were recovered. After nine months graft function was assessed by electrophysiological and anatomical methods.

Successful graft function was obtained in all animals.

Freeze-thawed skeletal muscle autografts are thus a convenient method of repairing the facial nerve in the CPA in sheep.

1 Glasby MA. Clinical Anatomy 1990;3:161-82.

A LABORATORY ASSESSMENT OF FIBRIN ADHESIVE AS A METHOD OF NERVE REPAIR JP Holland, BA Bell. Atkinson Morley's Hospital, Copse Hill, Wimbledon, London, UK

A technique to rejoin a severed facial nerve in the cerebellopontine angle after the removal of an acoustic neuroma using fibrin adhesive (Tisseel) to encase a primary facial nerve graft, with the sural nerve as donor, has been used with recovery of satisfactory facial function in $80 \%$ of patients. ${ }^{12}$

This paper describes a laboratory electrophysiological and histological assessment of nerve recovery after a Tisseel anastamosis. Fifteen rats had their left sciatic nerves severed under general anaesthetic and the nerve was reanastamosed by one of three methods: $12 / 0$ epineural nylon microsuture $(n=5)$, fibrin adhesive (Tisseel, $n=5)$, and cyanoacrylate adhesive $(n=5)$. After 100 days the rats underwent functional assessment on a climbing frame, nerve conduction studies, and histological assessment of the repaired nerve.

There were no significant differences in hind limb function or electrophysiological parameters (nerve conduction velocity, amplitude and duration of evoked potentials) between the three methods of nerve repair.

The considerable practical advantages of a tissue adhesive method of nerve repair are the ease and speed of the technique at the end of a long operation in a confined space and our preliminary results, which require histological verification, indicate that there are no advantages with the traditional epineural suture.

1 Walsh AR, Falci S, Weir NF, Schwartz $M$, Bell BA, Uttley D. $f$ Neurol Neurosurg Psychiatry 1990;53:448.

2 Bell BA, Weir N, Haglund MM. Congress of Neurological Surgeons Abstracts, 1993:129.
RECEIVER OPERATOR CHARACTERISTIC (ROC) CURVES: A NEW APPROACH TO THE ANALYSIS OF CEREBRAL PERFUSION PRESSURE AND INTRACRANIAL PERFUSION PRESSURE IN RELATION TO OUTCOME IN SEVERE HEAD INJURY

IR Chambers, MS Choksey, AD Mendelow. Regional Medical Physics Department and Department of Surgery (Neurosurgery), University of Newcastleupon-Tyne, UK

A method of analysing intracranial pressure (ICP) and cerebral perfusion pressure (CPP) data from severely head injured patients using receiver operator characteristic (ROC) curves is presented.

One hundred and eighty patients admitted with severe head injury were studied. For each patient, minimum CPP and maximum ICP values for any one hour interval during the recording were calculated. Outcome was determined with the Glasgow outcome score at six months. For CPP values from 30 to $130 \mathrm{~mm} \mathrm{Hg}$ and ICP values from 0 to $100 \mathrm{~mm} \mathrm{Hg}$ the sensitivity and specificity of that value being a predictor of an independent (good recovery or moderate disability) or poor (severely disabled, vegetative, or dead) outcome was established. Similar values were used for mortality. By plotting the sensitivity of each of these values against 1-specificity an ROC curve is produced.

The best curves were produced by adding the age of 40 into the criteria. In the over 40 group there was no association with CPP or ICP whereas in the under 40 age group the minimum number of "wrong" readings (false positives and false negatives) were at a CPP value of $54 \mathrm{~mm} \mathrm{Hg}$ and ICP of $36 \mathrm{~mm} \mathrm{Hg}$.

Values of ICP, CPP, and age have been identified as strong predictors of outcome; further analysis may yield other important thresholds that may be useful in the management of the severely head injured patient.

CEREBRAL HAEMODYNAMIC AND METABOLIC EFFECTS OF THE NON-COMPETITIVE NMDA ANTAGONIST CNS 1102 IN HUMANS WITH SEVERE HEAD INJURY

A Wagstaff, GM Teasdale, L Stewart, G Clifton. Department of Neuroanaesthesia, University Department of Neurosurgery, Institute of Neurological Sciences, Glasgow, UK and Department of
Neurosurgery, University of Texas, Houston, USA

Most head injured patients who subsequently die have evidence of ischaemia at postmortem; this is most often focal ischaemia associated with haematomas and contusions. The glutamate antagonists have 
proved very effective neuroprotective agents in animal models of focal ischaemia. CNS 1102 has a high affinity and selectivity for a binding site within the transmembrane ion channel associated with the NMDA receptors. Agents that bind to this site interfere with the function of the NMDA receptors by occluding the open channel, thereby preventing $\mathrm{NA}^{+}$and $\mathrm{CA}^{2+}$ ions from entering the cell. CNS 1102 has been shown to protect cultured brain cells against toxic concentrations of glutamate in vitro. In animal models of acute ischaemia (permanent and reversible middle cerebral artery occlusion) intravenous administration of CNS 1102 substantially reduced (by $40-70 \%$ ) the amount of damage. The first dose escalation and safety tolerability assessment of this agent in humans with severe head injury has been performed.

Patients who had sustained a severe head injury (Glasgow coma score 4-10) within the preceding 72 hours whose condition was stable and who were undergoing ventilation and intensive monitoring were given a single bolus of CNS 1102 followed by an infusion of the drug for four hours. The physiological parameters monitored were mean arterial blood pressure, intracranial pressure, cerebral perfusion pressure, jugular bulb oxygen saturation, ECG, temperature, and 21 channel EEG. Assessment of outcome is being made at six months using the Glasgow outcome score.

CNS 1102 was well tolerated by these patients. There were no adverse effects. Mean ICP and temperature fell during administration of higher doses of the drug and returned to the baseline within 12 hours; no other parameters changed significantly.

AN EXPERIMENTAL MODEL OF GLOBAL CEREBRAL ISCHAEMIA INDUCED BY PROFOUND HYPOTENSION BASED ON A COMPUTER DRIVEN CLOSED LOOP SYSTEM

P Smielewski, PJ Kirkpatrick, H Richards, JD Pickard. MRC Cambridge Centre for Brain Repair, Academic Neurosurgical Unit, Addenbrooke's NHS Trust, Cambridge, UK

Arterial hypotension is a recognised cause of reduced cerebral perfusion in several conditions including head injury. The aim of this study was to develop an experimental model, allowing the study of the cerebrovascular changes that accompany profound hypotension and the assessment of the effectiveness of brain protective agents. Reduction in arterial blood pressure was obtained by controlled withdrawal of arterial blood. The system consists of a computer driven pump capable of both infusing and withdrawing, an arterial blood pressure monitor, transcranial Doppler ultrasonograph set up for measurement of blood flow velocity in the basilar artery, and laser Doppler flowmeter for measurement of cortical microcirculation. The course of the experiments consisted of 30 minutes of baseline recording followed by another 30 minutes of deep hypotension and finally one hour postrecovery recording. The arterial blood pressure or transcranial Doppler blood flow velocity may be used as a closed loop control variable. Flow velocity control proved to be more reliable in maintaining a stable hypotensive state than when arterial blood pressure was used-the ratio of standard deviation to mean arterial blood pressure during hypotension was significantly lower when the flow velocity was used $(4.3 \%$ and $12 \%$ respectively, $\mathrm{p}<0.023)$.

After hypotension a hyperaemic response was seen and this was followed by a gradual decrease of all measured quantities to levels far below the baseline. These responses may be quantified and provide an experimental model for the assessment of an ischaemic insult.

EVENT RELATED POTENTIALS IN THE

ASSESSMENT OF TRAUMATIC COMA

N Kane, S Curry, S Butler, B Cummins, A Manara, $T$ Moss. Burden Neurological Institute and Frenchay Hospital, Bristol, UK

The prognostic value of multimodality evoked potentials (EPs) and event related potentials (ERPs) during coma (Glasgow coma score (GCS) $<8$ post-resuscitation) were investigated after severe traumatic brain injury (TBI).

Data were collected from 40 comatose patients with TBI (age range 1 to 80 years, mean 36; mean GCS 5.6). Recordings were performed in the first five days postinjury and repeated beyond the return of consciousness (defined as comprehension of simple verbal commands). Electrophysiological responses were recorded from 11 scalp electrodes with earlobe reference. Conduction times were measured for visual, auditory, and somatosensory short latency EPs, and long latency components of ERPs, generated by passive "oddball" paradigms. Patient outcome as determined by Glasgow outcome scale (GOS), in survivors between three and 12 months (mean seven), has been correlated with electrophysiological parameters using Pearson's coefficient.

Highly significant $(p<0.01)$ correlations exist between long latency ERPs components and GOS, together with some CT and clinical features (GCS, duration of coma, and PTA). Of the short latency EPs only somatosensory conduction time correlated significantly with outcome $(p<0.05)$. Unfortunately, due to variance of long latency responses in our controls absolute values cannot be relied upon as prognosticators. The presence of "mismatch negativity", an N2 component, predicted the return of consciousness ( $90 \%$ sensitivity) and preceded GCS changes. Its latency was the single best indicator of early outcome from coma $(r=0 \cdot 69)$.

1 Marshall LF, Marshall SB, Klauber MR, et al. f Neurosurg 1991;75(suppl):S14-S20.

INTRACRANIAL PRESSURE WAVEFORM INDICES CORRELATE WITH CEREBRAL PERFUSION PRESSURE BUT FAIL TO PREDICT EFFICACY OF MANNITOL THERAPY FOR RAISED ICP: A PROSPECTIVE STUDY IN HEAD INJURED PATIENTS

IR Piper, S Midgely, NM Dearden, FC Wong, MJ Souter, JD Miller. Department of Clinical Neurosciences, University of Edinburgh, Western General Hospital, Edinburgh, UK

It is known that the intracranial pressure waveform shape changes with cerebral perfusion pressure (CPP).

In a prospective study carried out over two years, ICP, arterial pressure (BP), and CPP waveform data were collected from 20 head injured patients during management in neurosurgical intensive care. On line minute by minute derivation of 12 indices of the ICP waveform shape based on both low and high frequency harmonic content were automatically collected by a purpose built waveform analysis system. Data collection was timed to coincide with minute by minute physiological data collection of heart rate, $\mathrm{BP}, \mathrm{ICP}, \mathrm{CPP}$, core temperature, arterial and jugular venous oxygen saturation $\left(\mathrm{SaO}_{2}, \mathrm{SjvO}_{2}\right)$, and end tidal $\mathrm{CO}_{2}$.

Data from 40 mannitol treatments (1856 minute by minute data points) were obtained from 12 patients (eight focal and four diffuse brain injuries) receiving treatment for raised ICP. There was no significant difference in physiological or ICP waveform parameters when either the pretreatment or post-treatment (point of maximum CPP) data were classified by type of head injury (focal/diffuse) or by the presence of hyperaemia (hyperaemic/non-hyperaemic). The pretreatment heart rate, however, was just significantly higher in the hyperaemic group ( $p<0.05$, ANOVA). To determine if ICP waveform parameters can predict the efficacy of mannitol treatment, pretreatment data were categorised by either treatment success or failure where success is defined as treatment which either reduces ICP below 20 or increases CPP above $60 \mathrm{~mm} \mathrm{Hg}$. With the exception of CPP pulse amplitude $(p=0.0074)$, which reflects the lower pretreatment CPP found in the treatment success group, there were no significant differences between treatment success/failure data for any pretreatment ICP waveform indices. When waveform indices pretreatment and post-treatment were compared four parameters were found to change significantly $(p<0.01)$ : ICP pulse amplitude, ICP distortion factor $k$, the pulsatility index of the CPP, and the amplitude transfer function (XFRaLF) of the fundamental harmonic. No waveform indices based solely on high frequency components changed significantly with CPP.

DETERMINATION OF CEREBRAL PERFUSION PRESSURE THRESHOLDS IN HEAD INJURED PATIENTS

FC Wong, IR Piper, JD Miller. Department of Clinical Neurosciences, University of Edinburgh, Western General Hospital, Edinburgh, UK

Transcranial doppler (TCD) monitoring can be useful in the management of cerebral perfusion pressure (CPP) in head injuries. This paper examines the potential uses of an on line computerised waveform analysis system of the blood pressure (BP), intracranial pressure (ICP), and TCD waveforms The main aim was to determine the parameters which may be useful in patients for defining the thresholds for adequate levels of CPP and the levels at which treatment is required.

Ten patients with head injuries and multiple physiological monitoring were studied in the intensive care unit. Simultaneous samples of BP, ICP, and TCD signals were obtained and their derived parameters from waveform analysis displayed on a personal computer in real time.

Waveform analysis data were obtained from the 10 patients during spontaneous 
and treatment induced periods of changing CPP. A relation between CPP and the pulsatility index of middle cerebral artery TCD waveform and between CPP and the fundamental transfer function from BP to ICP was seen in six patients that demonstrated a breakpoint near $70 \mathrm{~mm} \mathrm{Hg}$ of CPP.

The breakpoint could, however, differ by at least $10 \mathrm{~mm} \mathrm{Hg}$ between and within patients on different days.

Simultaneous monitoring of BP, ICP, and TCD signals can help to determine the lower limit above which patients' CPP should be maintained. This breakpoint limit can be determined by different parameters and can be used to "tailor" the CPP management thresholds to the individual patient.

A METHOD FOR CONTINUOUS INFUSION OF TROPHIC FACTORS INTO RAT SPINAL

SUBARACHNOID SPACE

EP Guazzo, RJM Franklin. University of Cambridge Departments of Neurosurgery and Clinical Veterinary Medicine and the MRC Cambridge Centre for Brain Repair, Cambridge, UK

The in vitro effect of trophic factors on cultures of CNS cells and their progenitors has been extensively studied. Intense interest is currently focused on investigating the possible role of trophic factors in augmenting spontaneous CNS repair and improving the efficacy of transplantation in the CNS. A method that delivers a continuous infusion of trophic factors into the rat spinal subarachnoid space has been developed, which facilitates the study of the effects of various trophic factors on repairing or transplanted spinal cord.

A three level laminectomy from $\mathrm{T} 13$ to L2 is performed on anaesthetised mature Sprague-Dawley rats. The preprimed, filled, and assembled osmotic minipump with connectors and fine silastic tube is positioned with the pump on the left side, secured in a subcutaneous position. The silastic tube enters the subarachnoid space and ends immediately rostral to the lesion. The continuous infusion required is specified by the selected pump (periods of seven to 14 days; rates of $0 \cdot 5-1 \mu \mathrm{l} /$ hour). Dye infusion studies, histological assessment, and postmortem examinations have been used to validate the method and its reliability. Preliminary experimental results comparing infusions of physiological saline and various trophic factors support the method's reliability and utility.

A reliable, validated method for the continuous infusion of trophic factors into the rat spinal subarachnoid space has been developed. It allows a broad range of strategies to be used in investigating the complex interactions between repairing or grafted spinal cord lesions and trophic factors.

PERIPHERAL NERVE REPAIR USING A FREEZETHAWED AUTOLOGOUS MUSCLE GRAFT

LM Myles, G Lawson, MA Glasby, JD Miller. Department of Clinical Neurosciences, Western General Hospital, Edinburgh and Department of Anatomy, University of Edinburgh, UK

After transection of a peripheral nerve, end to end suture is possible only if the length of damaged or lost nerve is very short. Nerve autografts have been used to fill such defects. Recent work has, however, shown coaxially aligned freeze-thawed muscle grafts to be a satisfactory means of repair in nerve defects of various species.

Groups of five sheep were used. A $3 \mathrm{~cm}$ muscle graft was placed in the median nerve using a standard microsurgical technique. At six months the regenerated nerve was dissected out and nerve conduction velocities recorded. Laser doppler blood flowmetry was also performed. Specimens of nerve were taken for morphometric analysis (fibre counts, fibre diameter spectrum, and myelin sheath thickness). The normal contralateral median nerve was used as a control.

On examination the muscle graft had become incorporated into the regenerated nerve in all five cases. Axonal regeneration through the muscle graft had been successful with absolute axon counts being normal or above normal in all five cases. Conduction velocities were slightly reduced in the regenerated nerve when compared with normal. This was reflected in a shift of the fibre diameter spectrum to the left and a reduction in myelin sheath thickness. Laser doppler blood flowmetry showed that the regenerated nerve had significantly poorer axial blood flow than normal nerve.

Freeze-thawed autologous muscle grafts are considerably easier to harvest and insert than cutaneous nerve grafts and seem to function well.

\section{NEUROPATHOLOGY OF SUBACUTE}

EXPERIMENTAL INTRACEREBRAL HAEMATOMA IR Whittle, JF Ironside. Departments of Clinical Neuroscience and Neuropathology Laboratory, Western General Hospital, Edinburgh, UK

Spontaneous intracerebral haematoma (ICH) is a common form of cerebrovascular accident that has a high morbidity. Many experimental studies have been pioneered and performed in Glasgow using a rodent caudatoputanimal ICH model. All these studies are acute and no longer term ( $>$ six hours) experiments were reported. To determine if there were compounds that are released by the haematoma or damaged perilesional brain, that potentiate perihaematoma brain dysfunction and damage, an ICH model with survival was required.

Adult male Wistar rats were given a general anaesthetic (intraperitoneal pentobarbitone $(30 \mathrm{mg} / \mathrm{kg})$ ), placed in a stereotaxic frame, and a burr hole drilled $3.5 \mathrm{~mm}$ lateral to the bregma point. Fresh blood was withdrawn from an arterial line in a syngeneic animal and $25 \mu \mathrm{l}$ stereotactically injected at a depth of $5.5 \mathrm{~mm}$ using a Hamilton syringe and a driving pressure of $90 \mathrm{~mm} \mathrm{Hg}$. The brain was sectioned and studied using conventional and immunocytochemical techniques. There were no subdural haematomas. Macroscopic sections of the brain showed deep punctuate haematomas with some spread into adjacent white matter tracts. There was no midline shift. Haematoxylin and eosin and luxol fast blue stains showed a patchy acute polymorphic response around the haematoma. The perilesional neuropil was vacuolated and pale suggesting significant oedema formation. There were significant changes in the neurons ranging from nuclear swelling to nuclear degeneration and pyknosis. These occurred in a centripetal, graded fashion around the haematoma. There were early signs of endothelial reaction, microglial infiltration, and astrogliosis. This simple model of experimental subacute ICH seems ideal for the study of experimental treatment that is designed to limit the size of perilesional infarction.

EFFECTS OF THIOPENTONE AND PROPOFOL ON MIDDLE CEREBRAL ARTERY BLOOD FLOW VELOCITIES IN ADULTS

SR Haynes, A Davidson, A Murray, AD Mendelow. Department of Anaesthesia, Newcastle General Hospital, UK

Twenty four adults (age 19-62 years) undergoing elective lumbar disc surgery were randomly selected to receive either thiopentone (group $\mathrm{T}$ ) or propofol (group P) for induction of anaesthesia. The data, documented both before induction of anaesthesia and when the eyelash reflex was lost after administration of the induction agent were: systolic, diastolic, and mean blood pressure, heart rate, and a five second transcranial Doppler trace of middle cerebral artery (MCA) flow velocity. Systolic, diastolic, and mean MCA flow velocities from both recordings were noted and the pulsatility index calculated. Comparisons were made between those given propofol and thiopentone both before and after drug administration. A p value $<0.01$ was taken as significant.

There was no significant difference between groups $T$ and $P$ in any documented parameter before induction of anaesthesia. The median (range) $P I$ in group $\mathrm{T}$ after induction was $0.605(0.381-1 \cdot 125)$, and in group $P 1 \cdot 115(0 \cdot 839-2 \cdot 100)$. This difference was highly significant, $p=0.0002$. This was the only significant difference between groups either before or after induction of anaesthesia.

Previous work ${ }^{1}$ has demonstrated that hemispherical blood flow correlates inversely with the pulsatility index in the MCA. There was no difference in haemodynamic parameters between groups $T$ and $P$ after induction of anaesthesia, therefore propofol reduces cerebral blood flow by a direct mechanism, and thiopentone has little effect.

1 Field LM, Dorrance DE, Krzeminska EK, Barzoum LZ. Br f Anaesth 1993;70:154-9.

EFFECT OF ISOFLURANE ON FREE RADICAL MEDIATED DAMAGE TO BRAIN TISSUE IN VITRO

AJ Davidson, D Mantle, AD Mendelow. Departments of Neurosurgery and Neurochemistry, Newcastle General Hospital, UK

An in vitro method was developed to investigate free radical induced damage in brain tissue preparations. This was used to explore the mechanism of putative cerebral protection obtained in vivo with the volatile anaesthetic isoflurane.

Postmortem human brain tissue was homogenised and separated by centrifugation into soluble and pellet fractions containing predominantly enzymatic and structural proteins respectively. After gassing with $5 \%$ isoflurane in either oxygen or nitrous oxide to selectively produce superoxide or hydroxyl radicals, the samples 
were sealed and exposed to a cobalt $60 \gamma$ irradiation source to facilitate radical generation.

The preparations were analysed by gel electrophoresis and the protein fractionation patterns compared with non-irradiated controls (not exposed to free radicals) to determine radical induced protein damage; there was no demonstrable protection in vitro by isoflurane from free radical induced protein damage shown in any of the assays analysed. It was not possible, therefore, to confirm the suggestion that the neuroprotective effect seen with isoflurane ${ }^{1}$ results from scavenging of free radical species as shown with other liquid anaesthetics. ${ }^{2}$ Isoflurane may effect protection by an alternative mechanism such as an action on membrane associated or calcium dependent proteases.

1 Sugaya T, Kitani Y. Br f Anaesth 1993;71: 247-52.

2 Murphy PG, Nyers DS, et al. Br f Anaesth 1992;68:613-8.

MONITORING THE HIGH RISK GROUP: CEREBRAL FUNCTION AND SOMATOSENSORY EVOKED POTENTIAL CHANGES DURING CAROTID ENDARTERECTOMY

DT Hope, V Orpe, LM Henderson, NJ Smith, S Rimmington. Department of Neurosurgery and The Department of Neurophysiology, University Hospital, Nottingham, UK

The publication of the European Carotid Surgery Trial ${ }^{1}$ and the North American Symptomatic Carotid Endarterectomy Trial $^{2}$ has resulted in patients with severe stenosis (greater than $70 \%$ ) coming to carotid endarterectomy. Many of these patients may have coexisting stenosis of the vertebral or contralateral carotid arteries, emphasising the need for careful intraoperative monitoring. Ninety three carotid endarterectomies have been performed (DTH) at University Hospital and all but two of these involved monitoring of both cerebral function analysis (CFAM), and somatosensory evoked potentials (SEPs) from the contralateral median nerve. We have adopted a policy of selective shunting based on changes in either CFAM or SEP. Changes in transcranial Doppler monitoring are demonstrated. Nineteen patients had either a critical stenosis or an occlusion of the vertebral or carotid artery of the opposite side. Results are shown in the table.

Overall 27 patients showed changes in either CFAM, or SEP, or both. Seven of these were in the "high risk" group. Seven patients had an "operative stroke" (within 24 hours). Six of these showed changes, three at carotid clamping, one before, and two later (after five minutes delay). In the whole series of 91 patients seven were shunted, based on monitoring changes. In conclusion, operative stroke is multifactorial but in this series it is considered that monitoring will be of increasing value in deciding when to shunt the high risk patient to pre- vent critical ischaemia during carotid endarterectomy.

1 European CST Group. Lancet 1991;337 $1235-43$

2 NASCET Collaborators. $N$ Engl $f$ Med 1991; 325:445-53.

EFFECTS OF ISCHAEMIA AND REPERFUSION ON THE PHARMACOLOGICAL RESPONSES OF SMALL CEREBRAL ARTERIES

R Laing, J Jakubowski, A Morice. Department of Neurosurgery, Royal Hallamshire Hospital, Sheffield, UK

Information about the effects of ischaemia or reperfusion on the viability and pharmacological responses of arteries distal to an occlusion would allow treatment to $\max$ imise blood flow through these arteries and so achieve optimum reperfusion of ischaemic brain after revascularisation. A period of four hours after arterial occlusion is the earliest clinically feasible time for the institution of thrombolytic treatment. Fifty adult Wistar rats were studied. An intravascular embolus was used to occlude the origin of the right middle cerebral artery (MCA). Ischaemia was confirmed by reduction in cerebral blood flow (CBF). The animals were divided into two groups. In 20 the right MCA was occluded for four hours and in a further 20 , four hours of ischaemia was followed by 90 minutes of reperfusion. Ten animals were used as controls. All animals were killed and both MCAs harvested. The arteries were mounted on a Mulvany myograph and the in vitro responses recorded at $\mathrm{mN}$ active tension. We measured responses to a range of pharmacological agents, both endothelium dependent and endothelium independent. Results have been analysed by paired $t$ test comparing each ischaemic and reperfused artery with its contralateral control. After acute ischaemia the MCA remains viable but the sensitivity of the arteries to some agents is reduced. Reperfusion reverses some of these changes but may exacerbate endothelial damage. The data show that ischaemia and reperfusion damages vascular function selectively. If these findings apply to human cerebral arteries treatment with vasodilators after revascularisation of ischaemic brain may result in preferential dilatation of normal vessels and vascular steal.

EFFECTS OF INTRATHECAL CALCITONIN GENE RELATED PEPTIDE ON ISCHAEMIC BRAIN INJURY

JP Holland, P Guyler, BA Bell. Atkinson Morley's Hospital, Copse Hill, Wimbledon, London, UK

Calcitonin gene related peptide (CGRP) is an endogenous vasodilating neuropeptide with a dense concentration around the cerebral arteries ${ }^{1}$; it plays a part in the control of vasomotor tone. Deficiency of the peptide has been implicated in the pathogenesis of delayed cerebral ischaemia after sub-

\begin{tabular}{lllll}
\hline & Stroke & Shunted & No stroke or shunt & Total \\
\hline Change in CFAM/SEP & $5(+1$ despite shunt $)$ & 7 & 15 & 27 \\
No change in CFAM/SEP & 1 & 0 & 63 & 64 \\
\hline
\end{tabular}

arachnoid haemorrhage (SAH). An intravenous infusion of CGRP in a rat model of middle cerebral artery (MCA) occlusion has been shown to reduce ischaemic neuronal damage by $58 \%$ and has identified the postischaemic therapeutic window at between one and two hours. ${ }^{2}$ The next stage of laboratory investigation of this peptide has been to examine its intrathecal efficacy.

Ten anaesthetised male Wistar rats were randomly assigned to receive either an intrathecal bolus of CGRP or saline, via a lumbar catheter. Each rat then underwent four hours of MCA occlusion measuring physiological parameters, MCA blood flow, and the volume of ischaemic brain injury.

Intrathecal CGRP increased ischaemic CBF by $110 \%(27 \cdot 4+2.3$ compared to $12.5+4.1 \mathrm{ml} / 100 \mathrm{~g} / \mathrm{min}, t=6.42, \mathrm{p}<$ 0.001 ) but the histological end point of the volume of ischaemic brain injury is still being evaluated.

The preliminary CBF data would suggest that the intrathecal route is efficacious for the administration of CGRP, but histological verification is required before establishing an intrathecal clinical trial of this peptide in SAH.

1 Edvinsson L, Ekman R, Jansen I, et al. Ann Neurol 1987;21:431-7.

2 Holland J, Sydserff S, Bell BA. $f$ Neurol

Neurosurg Psychiatry 1994 (in press).

ROMAN TREPANNING: THE SURGEONS, INSTRUMENTS, AND PATIENTS

R Jackson. British Museum, London, UK

As in many earlier and subsequent cultures the Romans resolved certain diseases of the head by trephination. The indication was sometimes, though probably not invariably, trauma, and the 1 st century $\mathrm{AD}$ author Cornelius Celsus describes in some detail the operation for a depressed skull fracture.

Celsus was neither a surgeon nor a physician but was evidently well acquainted with their methods. Although he divided medicine into dietetics, drugs, and surgery, he did not distinguish the practitioners, calling them all medicus. Galen, on the other hand, as well as being a prolific medical writer of the 2 nd century $\mathrm{AD}$, was a practitioner of both medicine and surgery. He implied that in Rome, at least, trephination and most operations of that kind were the preserve of those who called themselves surgeons (chirurgi), and archaeological finds tend to support that notion.

The archaeological evidence of trephination includes both surgical tools and perforated crania. The identifiable tools are restricted to diagnostic types made of inorganic materials, most striking of which are a few examples of the crown trephine, but among which are also chisels and forceps. The skeletal remains, sometimes equivocal, include both healed and unhealed trephinations.

CEREBRAL NEAR INFRARED SPECTROSCOPY IN ADULTS: CONTRIBUTION OF EXTRACEREBRAI TISSUE TO ATTENUATION CHANGES

TJ Germon, NM Kane, AR Manara, RJ Nelson. Departments of Neurosurgery and Anaesthetics, Frenchay Hospital, Bristol, UK

The capacity of near infrared (NIR) spectroscopy (INVOS 3100, Somanetics, Troy, 
Michigan, USA) was studied in regard to detecting and distinguishing between major changes in extracranial and cerebral oxygenation and blood flow as reflected by an estimate of regional cerebral oxygen saturation $\left(\mathrm{rSO}_{2}\right)$

In 12 healthy volunteers one minute of frontalis muscle exercise (which induces local muscle ischaemia beneath the site of application of the Invos sensor) resulted in a significant reduction in $\mathrm{rSO}_{2}$ from 73 (7) $\%$ to $68(6) \%(p<0.001)$. Inflation of a pneumatic tourniquet proximal to the sensor site (which produces circulatory standstill in the extracranial tissues, confirmed by laser Doppler flowmetry) resulted in a significant reduction in $\mathrm{rSO}_{2}$ from $72(6) \%$ to $59(7) \%(\mathrm{n}=8, \mathrm{p}<0.001)$. The induction of moderate systemic hypoxia under full physiological and neurological monitoring in the presence of stable scalp ischaemia reduced $\mathrm{rSO}_{2}$ further from 57 (4)\% to 41 (6) $\%$ and over this range there was a good linear correlation with $\mathrm{SpO}_{2}(\mathrm{r}=0 \cdot 811)$. In anaesthetised patients a $115 \%$ increase in middle cerebral artery (MCA) flow velocity induced by mild hypercapnia was accompanied by a $4 \cdot 1 \%$ increase in $\mathrm{rSO}_{2}$, but in the presence of scalp ischaemia this $\mathrm{rSO}_{2}$ response was lost.

These studies show that the Invos 3100 signal is sensitive to tissue oxygenation and can detect hypoxia deep to the scalp. There is, however, no evidence that it can reliably detect cerebral hyperaemia induced by hypercapnia and the problem of spatial resolution using this technology has to be further refined before this equipment can be adopted for routine clinical monitoring.

MECHANISMS OF DRUG RESISTANCE IN MALIGNANT GLIOMA

DGT Thomas, SM Ashmore, J Beith, A Sankar, JL Darling. Gough-Cooper Department of Neurological Surgery, Institute of Neurology, National Hospital for Neurology and Neurosurgery, Queen Square, London, UK

The biochemical events associated with drug resistance have been investigated in a panel of short term cultures derived from malignant glioma. The ability to repair DNA interstrand crosslinks (ISCs) was determined by alkaline elution and levels of alkyltransferase (AT) activity were measured enzymatically. The extent of ISCs correlated well with sensitivity to nitrosoureas, although a small number of cultures with no AT activity were still resistant to these drugs. When these cultures were assayed for sensitivity to vincristine and adriamycin, the patterns of cross resistance fell into three groups. All cultures that were resistant to both drugs were composed of large numbers of PgP positive cells, whereas sensitive cultures had few positive cells. Some cultures of intermediate sensitivity did not express PgP suggesting that alternative mechanisms of resistance were present in these cells. High resolution proton spectra were obtained from these cultures. The spectra shared common peaks producing a consistent metabolic profile; however, variation was seen in peaks at three positions, $1.46 \mathrm{ppm}, 3.55 \mathrm{ppm}$ and $3.7 \mathrm{ppm}$, which tentatively correspond to alanine, glycine, and a mixture of other amino acids. Cultures sensitive to nitrosoureas tended to have higher amounts of these three metabolites than resistant cultures. The results of this study indicate that the heterogeneity of malignant glioma extends to drug resistance at a molecular level and that studies involving proton spectroscopy are likely to be helpful in investigating drug resistance in glioma.

FURTHER IN VITRO STUDIES OF THE EFFECTS OF PHOTODYNAMIC TREATMENT IN MENINGIOMAS

TS Buxton, PV Marks. Royal Hallamshire Hospital, Sheffield and Leeds General Infirmary, Leeds, UK

Despite being benign CNS tumours, meningiomas are not always curable. Adjuvant treatments such as radiotherapy or hormone manipulation are unsatisfactory. Photodynamic treatment (PDT) involves the uptake of a photosensitising drug by tumour tissue and its subsequent activation by light and is active against meningiomas in vitro. ${ }^{1}$ In the rat glioma model, haematoporphyrin derivative (HPD) can be activated not only by red light but also by ionising radiation. ${ }^{2}$ An in vitro study of the effects of PDT using light and $\gamma$ rays against a variety of meningiomas was conducted. Meningioma cells established in culture and transferred to 96-well plates were incubated with HPD (concentrations $0 \cdot 125-20 \mu \mathrm{M}$ ) and irradiated with $630 \mathrm{~nm}$ (red) light and $\gamma$ ray $(60 \mathrm{CO})$ alone and in combination. The HPD supernatant was removed and fresh medium added. Twenty four hours after irradiation quantitative analysis of the tumoricidal effect was made by the MTT colorimetric assay.

Photodynamic treatment using HPD as a photosensitising drug showed dose dependent tumoricidal activity and the addition of $60 \mathrm{CO}$ to light in the presence of HPD produced a greater cytotoxic effect than light or $60 \mathrm{CO}$ irradiation alone. These experiments on in vitro meningioma cells suggest a radiosensitising effect of HPD. The findings may be of importance in the development of new adjuvant treatment for meningioma.

1 Marks PV, Furneaux C, Shivvakumar R. Br $\mathcal{f}$ Neurosurg 1992;6:327-32.

2 Kostron $\mathrm{H}$, Swartz MR, Miller DC et al. Cancer 1986;57:964-70.

ANATOMICOFUNCTIONAI. CORRELATES OF LANGUAGE DISORDERS IN DOMINANT HEMISPHERIC NEOPLASMS: A PROSPECTIVE STUDY

A-M Thomson, $\mathbf{R}$ Taylor, $\mathrm{R}$ Sellar, D Fraser, I Whittle. Department of Clinical Neurosciences, Western General Hospital, Edinburgh, UK

Studies of the topographical relation between focal brain infarction and language disorders have led to current understanding of anatomicofunctional relations. As there are fundamental differences between the pathophysiology of brain tumours and stroke this prospective study was designed to examine correlations between the location of dominant hemispheric brain tumours and language dysfunction. The influence of lesion volume on dysphasia was also studied.

Language dysfunction was measured by standardised dysphasia tests: the western aphasia battery and Boston naming test. Tumour location was assessed indepen- dently by a neuroradiologist and neurosurgeon from CT and MR. Six neuroanatomical regions were identified-frontal temporal, occipital, Broca's, lateral temporoparietal, and central. Tumour volume was calculated with a computer algorithm.

Thirty four patients have been assessed so far. The tumour cohort included glioblastoma (12), anaplastic astrocytoma (nine), meningiomas (six), metastatic carcinomas (three), oligoastrocytomas (two), and one each pineal and pituitary tumours. Except for the occipital pole the tumours were randomly distributed throughout the six regions, often straddling several regions. Tumour volumes ranged from 8 to 210 $\mathrm{cm} .^{3}$ Test results showed that only very weak correlations existed between tumour location or size of tumour and type and severity of language impairment. The relevance of tumour neuropathology will be analysed when patient groups are larger.

The preliminary results of this prospective study suggest that there are profound differences between the described patterns of aphasia in patients with strokes and brain tumours at similar dominant hemispheric locations.

EXPERIMENTAL IMPLANTATION GLIOMA USING THE A15A5 CELL LINE

G Malcolm, PA Kelly, IM Ritchie, JW Ironside, IR Whittle. Departments of Clinical Neurosciences and Neuropathology Laboratory, Western General Hospital, Edinburgh, UK

To experimentally study aspects of peritumoral biology and to establish an in vivo test system to evaluate aspects of glioma treatment the rodent A15A5 glioma implantation model, which has previously been partly characterised in BIDX rats, was established. The neuropathology, growth patterns, and cerebrovascular features of this model were compared with the $\mathrm{C} 6$ model.

The A15A5 glioma cell line was kindly provided by $\mathrm{G}$ Pilkington. It was cultured at $37^{\circ} \mathrm{C}, 5 \% \mathrm{CO}_{2}$ in Hams $\mathrm{F}-12$ media with added glutamine, fungizon, penicillin, and streptomycin. When confluent the cells were washed in Dulbecco's buffered saline, disaggregated with $1 \%$ buffered trypsin, resuspended in Hams F-12 medium, and centrifuged $(\times 1000$ for five minutes $)$ $10-20 \mu 1$ of cell suspension $(3 \times 106$ cells $)$ was then stereotactically injected into the caudatoputamen nucleus (bregma- $1 \mathrm{~mm}$, $4 \mathrm{~mm}$ lateral, $5 \mathrm{~mm}$ depth) of anaesthetised adult Wistar rats (weight $282-450 \mathrm{~g}$ ) using a Hamilton syringe. Brains were removed at four to five day intervals after implantation (range seven to 20 days) using perfusion fixation with buffered glutaraldehyde $(2.5 \%)$ Tumour neuropathology was characterised by routine and immunocytochemical techniques. Assessment of perilesional blood flow, blood-brain barrier function and regional glucose metabolism was performed between days 12 and 20 using standard quantitative autoradiographic techniques.

With an innoculum of $3 \times 106$ cells $75 \%$ of rats $(n=20)$ developed tumours. Viability of non-implanted cells 90 minutes after completion of all implantations was greater than $93 \%$. Tumour growth caused no apparent neurological manifestations and no deaths before day 20 . Volumetric 
analysis showed tumour volumes varying from 45 to $110 \mathrm{~mm}^{3}$ with growth patterns being fairly reproducible at specific times after implantation. Blood-tumour transfer of $\left[{ }^{14} \mathrm{C}\right] \mathrm{AIB}$ was $62(7) \mathrm{ml} / \mathrm{g} / \mathrm{min} \times 10$; there was, however, a 10-fold region to region difference in capillary permeability. Tumour blood flow varied from 142 to 94 $\mathrm{ml} / 100 \mathrm{~g} / \mathrm{min}$. Overall in our centre the A15A5 model seems a much more reliable and predictable model of experimental rodent brain than the $\mathrm{C} 6$ model. Permeability and blood flow characteristics are, however, similar.

ASSESSMENT OF ALTERED CEREBRAL METABOLISM ASSOCIATED WITH ARTERIOVENOUS MALFORMATIONS USING IN VIVO PROTON MR IMAGING AND WATER SUPPRESSED LOCALISED PROTON SPECTROSCOPY

CM Moore, OM Redmond, PJ Gilligan, J McEnri, C Georgopolus, S O'Laoire, JP Stack, JT Ennis. Institute of Radiological Sciences and Department of Neurosurgery, Mater Private Hospital, Eccles St, Dublin, Ireland

Spectra were acquired in tissue adjacent and contralateral to a supratentorial arteriovenous malformation (AVM) from patients $(n=13)$ with epilepsy and medium to high flow AVMs. Control spectra were also acquired.

Patients were divided into: $(a)$ those that exhibited no signal enhancement and $(b)$ those that did, in tissue adjacent to the AVM, on T2 weighted spin echo images. $\mathrm{N}$-Acetyl aspartate (NAA), creatine (phosphocreatine) (PCr); and choline (Cho) peak areas were calculated. NAA is a neuronal marker: reduced NAA may be attributed to ischaemia; $\mathrm{Cr}(\mathrm{PCr})$ is a high energy metabolite, and Cho is involved in phospholipid metabolism. NAA in adjacent tissue was lower and Cho higher in all patients than in controls.

For type $a$ : The range of NAA values was larger than for class $b$ or controls. The signal enhancement is possibly a result of vasogenic oedema or gliosis because both sub-acute and long-standing AVMs were included in the study; thus explaining the larger range of metabolite values. Alternatively the larger range may be a result of medium flow AVMs not producing a severe nutritional deficit in the adjacent tissue for some patients.

For type $b$ : NAA was lower and Cho higher in adjacent tissue compared with controls. Lack of signal enhancement may be due to a signal void as a result of very high flow AVMs; the AVMs producing an ischaemic region yielding tissue necrosis and fatty acid metabolism.

MANAGEMENT OF ANEURYSMAL

SUBARACHNOID HAEMORRHAGE IN THE NORTH WESTERN REGION

RH Lye, L Hunt, K Chishti, K Hussain, R Page, P Pandya, H Parekh, D Rawluk, D Sandeman, R Strachan. Neurosurgical Centres at Hope, Manchester Royal Infirmary, North Manchester General, and Royal Preston Hospitals

During 1989-92 the four centres in the North Western Region audited the outcome in 466 adult patients with aneurysmal subarachnoid haemorrhage. The recruitment

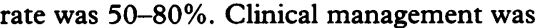
autonomous. Among 172 (37\%) men and $294(63 \%)$ women there was a female preponderance in the $>50$ year age groups. On admission $79(17 \%)$ patients were hypertensive; 60 (13\%) experienced headache one week before ictus. Multiple aneurysms occurred in $126(27 \%)$ patients.

Within 96 hours of ictus $60-80 \%$ of patients commenced nimodipine treatment and $86-96 \%$ of patients were WFNS grades I/II at operation, with variation according to centre and year of recruitment. The table summarises other findings.

Analysis suggests scope for improvement in some centres but results should be interpreted with caution.

A SIMPLE HISTOLOGICAL METHOD FOR THE EARLY DETECTION OF NEURONAL DAMAGE IN VIVO

GJC Wilde, L Sundstrom, J Mepham, F Iannotti. Clinical Neurological Sciences, University of Southampton, Southampton General Hospital, Southampton, UK

Propidium iodide in vitro is a marker for cell death: it crosses damaged cell membranes, binds to DNA, and fluoresces when excited by light passed through a rhodamine filter. The utility of propidium iodide as an in vivo marker of cell death has been investigated. Simultaneous injections of propidium iodide and kainic acid were made into the left cerebral ventricle of rats ${ }^{1}$ and the sensitivity of this new method was compared with that of thionine, a standard histological stain. One hour after the administration of kainic acid there were no apparent signs of cell loss, or even cell damage, using thionin. Propidium iodide, however, clearly showed that a small population of neurons within the CA3 were dying, each individual cell being easily identified by its cytoplasmic fluorescence. Six hours after the injection conventional histology did not show cell loss, although pyknosis was evi-

\begin{tabular}{|c|c|c|c|c|c|c|c|c|c|}
\hline \multirow[b]{2}{*}{ Centre } & \multirow[b]{2}{*}{$n$} & \multirow{2}{*}{$\begin{array}{l}\text { Admit } \\
N S U \\
<4 \text { days } \\
(\%)\end{array}$} & \multirow{2}{*}{$\begin{array}{l}\text { WFNS } \\
I \text { and II on } \\
\text { transfer } \\
(\%)\end{array}$} & \multirow{2}{*}{$\begin{array}{l}\text { Op } \\
\text { rate } \\
(\%)\end{array}$} & \multirow[b]{2}{*}{$\begin{array}{l}\text { Total no } \\
\text { operated }\end{array}$} & \multicolumn{2}{|l|}{ Op timing } & \multicolumn{2}{|c|}{ Outcome GOS } \\
\hline & & & & & & $\begin{array}{l}<4 \text { days } \\
(\%)\end{array}$ & $\begin{array}{l}>7 \text { days } \\
(\%)\end{array}$ & $\begin{array}{l}\text { ( } 3 \text { and } 4) \\
(\%)\end{array}$ & $\begin{array}{l}5 \\
5 \\
(\%)\end{array}$ \\
\hline $\begin{array}{l}\text { A } \\
\text { B } \\
\text { C } \\
\text { D } \\
\text { All }\end{array}$ & $\begin{array}{r}111 \\
143 \\
117 \\
95 \\
466\end{array}$ & $\begin{array}{l}87 \\
88 \\
88 \\
95 \\
89\end{array}$ & $\begin{array}{l}76 \\
77 \\
77 \\
89 \\
79\end{array}$ & $\begin{array}{l}82 \\
76 \\
88 \\
81 \\
82\end{array}$ & $\begin{array}{r}91 \\
109 \\
103 \\
77 \\
380\end{array}$ & $\begin{array}{r}50 \\
31 \\
17 \\
3 \\
27\end{array}$ & $\begin{array}{l}25 \\
41 \\
49 \\
86 \\
51\end{array}$ & $\begin{array}{r}14 \\
19 \\
14 \\
8 \\
14\end{array}$ & $\begin{array}{r}7 \\
13 \\
13 \\
2 \\
9\end{array}$ \\
\hline
\end{tabular}

Analysis suggests scope for improvement in some centres but results should be interpreted with caution. dent. By contrast the propidium iodide clearly showed that most neurons within the CA3 were dying; this was evident by the presence of brightly fluorescent cells within the subfield. Thus propidium iodide given in vivo was reliable as an early indicator of cell damage, anticipating ultimate cell loss before conventional histology.

1 Nadler JV, Perry BW, Cotman CW. Nature 1978;271:676-7.

EFFECTS OF DECREASING CEREBRAL PERFUSION PRESSURE ON PULSATILITY OF CEREBRAL BLOOD FLOW VELOCITY: A MODELLING STUDY

M Czosnyka, S Piechnik, H Richards, P Smielewski, $\mathbf{P}$ Kirkpatrick, JD Pickard. University of Cambridge Neurosurgical Unit, Addenbrooke's Hospital, Cambridge, UK and Warsaw University of Technology, Poland

An attempt was made to evaluate a mathematical model able to simulate the dynamic pressure flow interactions in two major pathways of fluid circulation through the intracranial space: blood and CSF. The study was aimed at the interpretation of clinical and experimental data showing an increase in pulsatility of cerebral blood flow velocity as cerebral perfusion pressure (CPP) decreases either as a result of intracranial hypertension or systemic hypotension. The analysis of phenomena taking place during intracranial hypertension caused by intracranial pressure (ICP) plateau waves was performed on the basis of computer recorded ICP, arterial blood pressure (ABP) and transcranial Doppler blood flow velocity (FV) from monitoring of five head injured patients. Systemic hypotension was studied using FV and ABP waveforms recorded during experimental gradual haemorrhage in 10 New Zealand white rabbits. Clinical data showed that above a certain ICP level corresponding to averaged mean CPP of $45 \mathrm{~mm} \mathrm{Hg}$ and averaged diastolic CPP of $35 \mathrm{~mm} \mathrm{Hg}$ (range from 30 to $43 \mathrm{~mm} \mathrm{Hg}$ ), diastolic $\mathrm{FV}$ started to decrease. The resulting increase in the systolic-diastolic distance of FV waveform was paralleled with reduction of the mean FV and an increase in the pulsatility index. Model analysis showed that the divergent behaviour of the systolic and diastolic FVs was initiated at diastolic CPP corresponding to the autoregulatory limit. Similarly, in analysis of experimental recordings, below average diastolic ABP of $55 \mathrm{~mm} \mathrm{Hg}$ (range from 65-44) diastolic flow velocity started to decrease and systolic flow velocity was stable. Systolic flow velocity started to fall when systolic ABP fell below $53 \mathrm{~mm} \mathrm{Hg}$.

In both cases the divergent behaviour of systolic and diastolic FV was caused by a wide gap between systolic and diastolic CPP. When diastolic CPP fell below the lower limit of autoregulation, diastolic FV decreased, mean FV started to decline smoothly, and systolic FV was stable. When systolic CPP fell below the lower autoregulatory threshold, the process of transition between completely intact and exhausted autoregulatory reserve was terminated. This transient zone, characterised by gradual divergence between systolic and diastolic $\mathrm{FV}$, increase in FV pulse amplitude, and pulsatility indices, is equal to the peak to peak. 